\title{
A Novel Model for Lithium-ion Battery Aging Quantitative Analysis Based on Pseudo Two-Dimension Expressions
}

\author{
Yizhao Gao ${ }^{1}$, Xi Zhang ${ }^{1 *}$,Jun Yang ${ }^{1}$, Bangjun Guo ${ }^{1}$, Xuan Zhou ${ }^{2}$ \\ ${ }^{1}$ School of Mechanical Engineering, Shanghai Jiao Tong University, Shanghai, China \\ ${ }^{2}$ Department of Electrical and Computer Engineering, Kettering University, Flint, USA \\ *E-mail: gaoyizhao@sjtu.edu.cn; braver1980@sjtu.edu.cn; dreamyang@ @jtu.edu.cn, \\ jasonguob@gmail.com
}

doi: $10.20964 / 2019.04 .05$

Received: 30 August 2018 / Accepted: 23 January 2019 / Published: 10 March 2019

\begin{abstract}
This paper proposes a novel model for lithium-ion battery aging quantitative analysis considering side reactions. The model is integrated with the transfer function type derived from a pseudo two-dimensional (P2D) model. The capacity fade, solid electrolyte interphase (SEI) growth and deposited layer growth led by the side reactions are selected as degradation representatives. The quantitative relationship between the Li ion concentration and fore-mentioned degradation parameters is established using some mathematical methods e.g., the Laplace transform and the Pade approximation, etc. to somehow indicate the battery aging mechanism. To observe the battery aging, scanning electron microscopy (SEM) and transmission electron microscopy (TEM) were employed to quantify the deposited layer and SEI thickness, respectively. The simulated and experimental results verified the correctness and validity of the proposed model.
\end{abstract}

Keywords: Lithium-ion battery, Side reaction, SEI growth.

\section{FULL TEXT}

(C) 2019 The Authors. Published by ESG (www.electrochemsci.org). This article is an open access article distributed under the terms and conditions of the Creative Commons Attribution license (http://creativecommons.org/licenses/by/4.0/). 